ARTICLE

\title{
Proline metabolism supports metastasis formation and could be inhibited to selectively target metastasizing cancer cells
}

Ilaria Elia ${ }^{1,2}$, Dorien Broekaert ${ }^{1,2}$, Stefan Christen ${ }^{1,2}$, Ruben Boon ${ }^{3}$, Enrico Radaelli ${ }^{4}$, Martin F. Orth ${ }^{5}$, Catherine Verfaillie ${ }^{3}$, Thomas G.P. Grünewald ${ }^{5} \&$ Sarah-Maria Fendt ${ }^{1,2}$

Metastases are the leading cause of mortality in patients with cancer. Metastasis formation requires cancer cells to adapt their cellular phenotype. However, how metabolism supports this adaptation of cancer cells is poorly defined. We use 2D versus 3D cultivation to induce a shift in the cellular phenotype of breast cancer cells. We discover that proline catabolism via proline dehydrogenase (Prodh) supports growth of breast cancer cells in 3D culture. Subsequently, we link proline catabolism to in vivo metastasis formation. In particular, we find that $P R O D H$ expression and proline catabolism is increased in metastases compared to primary breast cancers of patients and mice. Moreover, inhibiting Prodh is sufficient to impair formation of lung metastases in the orthotopic 4T1 and EMT6.5 mouse models, without adverse effects on healthy tissue and organ function. In conclusion, we discover that Prodh is a potential drug target for inhibiting metastasis formation.

\footnotetext{
${ }^{1}$ Laboratory of Cellular Metabolism and Metabolic Regulation, VIB Center for Cancer Biology, VIB, Herestraat 49, Leuven 3000, Belgium. ${ }^{2}$ Laboratory of Cellular Metabolism and Metabolic Regulation, Department of Oncology, KU Leuven and Leuven Cancer Institute (LKI), Herestraat 49, Leuven 3000, Belgium. ${ }^{3}$ Stem Cell Institute, KU Leuven, Herestraat 49, Leuven 3000, Belgium. ${ }^{4}$ Center for the Biology of Disease, VIB Leuven and Center for Human Genetics, KU Leuven, Herestraat 49, Leuven 3000, Belgium. ${ }^{5}$ Max-Eder Research Group for Pediatric Sarcoma Biology, Institute of Pathology, LMU Munich, Thalkirchner Strasse 36, Munich 80337, Germany. Correspondence and requests for materials should be addressed to S.-M.F. (email: sarah-maria.fendt@kuleuven.vib.be).
} 
M etabolic reprogramming is recognized as a hallmark of cancer cells that supports cancer growth ${ }^{1}$. Investigating how metabolism supports cancer growth resulted in several metabolism-based drugs that are now in clinical $\mathrm{trial}^{2}$. Yet, some cancer cells within a progressing tumour acquire additional cellular phenotypes, such as motility, invasion, survival and colonization capacity, which are supporting cancer progression towards metastasis formation ${ }^{3}$. Thus, identifying how metabolism supports shifts in the cancer cell phenotype that can contribute to metastasis formation has the potential to identify innovative drug targets against cancer progression.

Ninety percent of all cancer deaths are caused by metastases in distant organs ${ }^{4}$. The metastasis formation cascade consists of several stages ${ }^{5,6}$ : First, cancer cells disseminating from the primary tumour invade the surrounding tissue and intravasate into the circulation. Next, cancer cells infiltrate and colonize a distant organ. At this stage, cancer cells can remain dormant or in a stable micrometastasis state for weeks to years, but eventually they will grow and form macrometastases, which results in established secondary tumours. Several studies have focussed on metabolic reprogramming during the early steps of metastasis formation, when cancer cells disseminate from the primary tumour, invade the surrounding tissue and survive in the circulation $^{7-9}$. From a clinical perspective, however, the later steps in metastasis formation are of specific interest, because patients often present to the clinics when cancer cells have already infiltrated a distant organ ${ }^{10,11}$. Yet, our knowledge on how metabolism supports cancer cell survival and colonization of a distant organ is limited to a few studies $7,12,13$.

Here we address the questions how metabolism supports phenotypic shifts in breast cancer cells and to which extent inhibiting these changes in metabolism can counteract in vivo lung metastasis formation. In particular, we enforce a phenotypic shift in breast cancer cells by cultivating them either as monolayer in two-dimensional (2D) culture or as spheroids in threedimensional (3D) culture. Next, we identify differences in the cellular metabolism of cells cultured in $2 \mathrm{D}$ versus $3 \mathrm{D}$ conditions. Finally, we investigate whether inhibiting the identified metabolic differences impairs in vivo metastasis formation without adverse effect on healthy tissue and organ function in mice. We discover that breast cancer cells grown in spheroids (3D) compared to attached monolayers (2D) increase proline catabolism via the enzyme proline dehydrogenase (Prodh). Inhibiting Prodh activity results in impaired spheroidal growth and in a dose-dependent decrease in lung metastasis formation in two mouse models. Pharmacological Prodh inhibition has no adverse effects in vitro on non-transformed mammary epithelial cells and in vivo on healthy tissue and organ function of mice. Thus we identify Prodh as promising drug target against breast cancer-derived metastasis formation.

\section{Results}

Proline catabolism distinguishes 2D from 3D growth. We used transformed human MCF10A H-Ras ${ }^{12}$ mammary epithelial cells to study phenotypic shifts induced by $2 \mathrm{D}$ versus $3 \mathrm{D}$ cultivation. This cell line has been used to study gene expression as well as signalling pathway activity during spheroidal growth ${ }^{14,15}$. MCF10A H-Ras V12 cells were derived from immortalized and non-tumorigenic breast epithelial cell line MCF10A (ref. 16). These cells were transduced with the oncogenic driver H-Ras ${ }^{\mathrm{V} 12}$, which is of relevance to the human breast cancer situation, since $50 \%$ of the human breast cancers display increased H-Ras activity 17 . To achieve spheroidal growth, we cultured MCF10A H-Ras ${ }^{12}$ cells on soft-agar coated plates in DMEM-F12 media (Supplementary Fig. 1). To compare metabolism during spheroidal and attached monolayer growth, we applied ${ }^{13} \mathrm{C}$ tracer analysis ${ }^{18}$. Since this method has so far not been used in soft-agar cultures, we tested the suitability of four different metabolite extraction methods ${ }^{19-22}$. We evaluated metabolite recovery, cellular energy charge and cellular protein recovery resulting from the four tested methods. We found that methanolchloroform extraction combined with a mechanical disruption of the spheroids is well suited to extract metabolites from spheroids cultured on soft-agar (Supplementary Fig. 2). Consequently, we performed all our subsequent experiments using this method.

Cancer cells growing either in attached monolayers (2D) or spheroids (3D) consume substantial amounts of the nutrients glucose and glutamine $2,23,24$. Yet, it remains elusive whether these nutrients are used to fuel metabolite production similarly in both culture conditions. Thus, we tested the contribution of both nutrients to organic and amino acid production in MCF10A H-Ras ${ }^{\mathrm{V} 12}$ in both conditions using ${ }^{13} \mathrm{C}_{6}$-glucose and ${ }^{13} \mathrm{C}_{5}$-glutamine. Interestingly, the most pronounced change observed was a decrease in the ${ }^{13} \mathrm{C}$ contribution from both nutrients to the production of the amino acid proline in MCF10A H-Ras ${ }^{\mathrm{V} 12}$ (Fig. 1a,b and Supplementary Fig. 3), which indicates that these cells switch to a different proline metabolism when grown in spheroids.

To validate whether proline catabolism or biosynthesis are different in MCF10A H-Ras ${ }^{\mathrm{V} 12}$ cells during spheroidal growth, we quantified proline uptake, intracellular proline levels and the expression of $P R O D H$ and pyrroline-5-carboxylate dehydrogenase $(P 5 C D H)$ (proline catabolism), as well as pyrroline-5carboxylate synthase (P5CS) and pyrroline-5-carboxylate reductase 1 (PYCR1) (proline biosynthesis) in both growth conditions. We found that cells consumed proline from the media only during spheroidal growth, while the same cells secreted proline to the media during attached monolayer growth (Fig. 1c). In accordance, intracellular proline levels were lower in cells grown in spheroids than the same cells grown in monolayer (Fig. 1d). This suggested that cells grown in spheroids require more proline, because spheroidal growth might result in proline catabolism. Indeed, the expression of the proline catabolism enzyme PRODH was significantly increased by approximately $300 \%$ in MCF10A H-Ras ${ }^{V 12}$ cells during spheroidal growth $(P \leq 0.01$, two-tailed unpaired Student's $T$-test) (Fig. 1e), while the expression level of $P 5 C D H$ and that of the proline biosynthesis genes P5CS and PYCR1 remained unchanged (Fig. 1e). Together, these data provide evidence that proline catabolism distinguishes spheroidal (3D) from attached (2D) growth in MCF10A H-Ras ${ }^{v 12}$ cells.

Proline catabolism via Prodh supports 3D growth. To investigate whether proline catabolism is required for spheroidal growth of MCF10A H-Ras ${ }^{\mathrm{V} 12}$ cells, we employed a genetic and catalytic inhibition of Prodh. To this end, we generated a doxycycline inducible CRISPR interference knockdown of $P R O D H$ in MCF10A H-Ras ${ }^{\mathrm{V} 12}$ cells, which had an efficiency of $96 \%$ (Supplementary Fig. 4a). Strikingly, knockdown of PRODH impaired spheroidal growth of MCF10A H-Ras ${ }^{\mathrm{V} 12}$ by approximately $70 \%$ based on spheroid size (Fig. 2a).

We next tested whether inhibition of Prodh activity could elicit a similar effect on spheroidal growth by using the compound L-tetrahydro-2-furoic acid (L-THFA), which was reported to inhibit Prodh activity in 2D cultures ${ }^{25}$. Since increased Prodh activity results in decreased intracellular levels of proline (Fig. 1d), we argued that L-THFA treatment should increase intracellular proline levels. Consistently, we found that L-THFA treatment increased intracellular proline levels in MCF10A H-Ras ${ }^{\text {V12 }}$ spheroids (Supplementary Fig. $4 \mathrm{~b}$ ) indicating that the compound effectively inhibits Prodh activity. Similar to the 
a

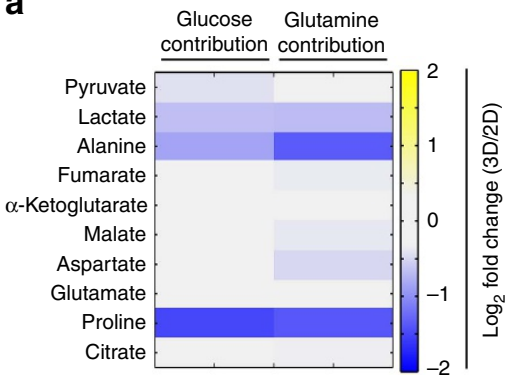

c

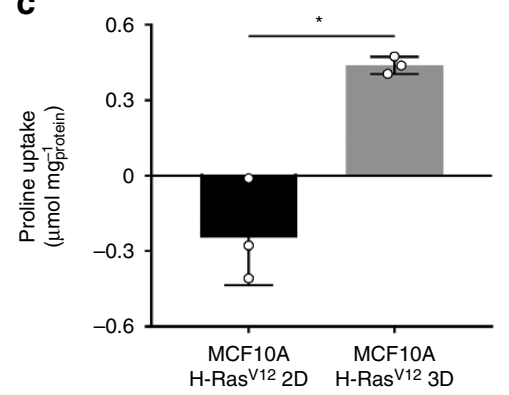

b

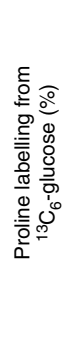

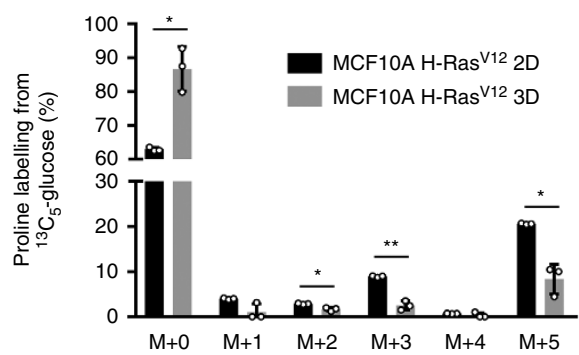

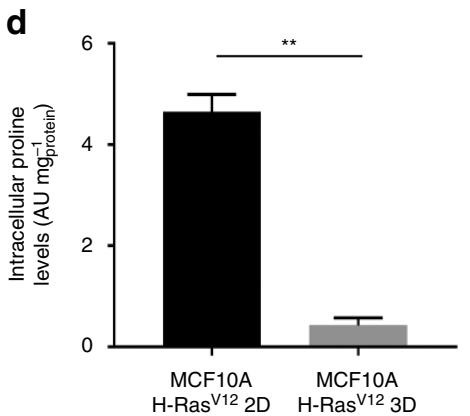

e

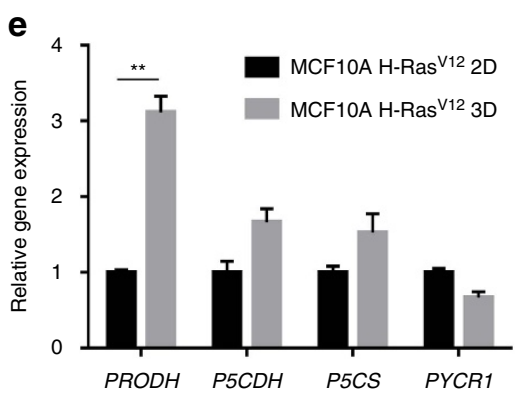

Figure 1 | Proline catabolism distinguishes 3D from 2D growth. (a) Total contribution of ${ }^{13} \mathrm{C}_{6}$-glucose and ${ }^{13} \mathrm{C}_{5}$-glutamine to different organic acids and amino acids in MCF10A H-Ras V12 spheroids (3D) versus attached cells (2D). Changes in total contribution $>25 \%$ with a $P$ value $\leq 0.05$ are depicted. (b) Proline labelling from ${ }^{13} \mathrm{C}_{6}$-glucose and ${ }^{13} \mathrm{C}_{5}$-glutamine in MCF10A H-Ras V12 spheroids (3D) versus attached cells (2D). (c) Proline uptake in MCF10A $\mathrm{H}$-Ras ${ }^{\mathrm{V} 12}$ spheroids (3D) versus attached cells (2D). (d) Intracellular levels of proline in MCF10A H-Ras V12 spheroids (3D) versus attached cells (2D). (e) Relative expression of proline catabolism (PRODH and P5CDH) and biosynthesis (P5CS and PYCR1) genes in MCF10A H-Ras V12 spheroids (3D) versus attached cells (2D) measured by qRT-PCR. The number of biological replicates for each experiment was $n \geq 3$. All error bars represent s.d. Two-tailed unpaired Student's $T$-test was performed. ${ }^{\star} P \leq 0.05 ;{ }^{\star \star} P \leq 0.01$.

PRODH knockdown, L-THFA treatment reduced the spheroid size of MCF10A H-Ras ${ }^{\mathrm{V} 12}$ spheroid growth by approximately 75\% (Fig. 2b). Collectively, these data show that Prodh activity is required for optimal spheroidal growth of MCF10A H-Ras v12 cells and suggested that Prodh may serve as a drug target.

Prodh activity depends on P5C recycling during 3D growth. Subsequently, we investigated whether proline withdrawal from the media can impair spheroidal growth. Surprisingly, we found that proline removal from the media had no effect on the growth of MCF10A H-Ras ${ }^{\mathrm{V} 12}$ spheroids (Fig. 3a).

Interestingly, Prodh activity yields the metabolite pyrroline 5-carboxylic acid (P5C), which can be recycled to proline via the NADPH consuming proline biosynthesis enzyme $\mathrm{Pycr}^{26}$. Thus, we hypothesized that $\mathrm{P} 5 \mathrm{C}$ recycling to proline via Pycr sustains Prodh activity. Consistently, we found that the NADPH to $\mathrm{NADP}^{+}$ratio was decreased during spheroidal growth (Fig. 3b).

To further confirm the compensatory role of $\mathrm{P} 5 \mathrm{C}$ recycling, we knocked down enzymes involved in proline recycling (PYCR1, PYCR2, PYCR3) (Supplementary Fig. 4c). Removal of proline from the media in combination with knockdown of PYCR1 strongly impaired spheroidal growth of MCF10A H-Ras ${ }^{\mathrm{V} 12}$ (Supplementary Fig. 4d). This implied that PYCR1 is the predominant PYCRx isoform generating proline from P5C in MCF10A H-Ras 12 spheroids. In line with the hypothesis that $\mathrm{P} 5 \mathrm{C}$ recycling is important for sustaining Prodh activity, we found that PYCR1 knockdown in MCF10A H-Ras ${ }^{\mathrm{V} 12}$ cells impaired spheroid growth by $73 \%$, despite proline availability in the media (Fig. 3c,d). In agreement with a decreased Prodh activity in PYCR1 knockdown cells, we observed that proline levels increased compared to control spheroids (Fig. 3e). Notably, while spheroidal growth of PYCR1 knockdown cells was decreased, the remaining spheroids additionally showed increased aggregation, which indicates an additional function of Pycrl that is independent of sustaining Prodh activity. This observation is in line with the finding that Pycr is involved in nucleotide and endoplasmic reticulum homeostasis $^{26,27}$. Taken together, our data show that Prodh activity during spheroidal growth is sustained by P5C recycling via Pycr1.

Prodh activity generates ATP during 3D growth. It was shown previously that Prodh activity can contribute to mitochondrial energy production by feeding electrons into the electron transport chain $^{28}$. To investigate whether proline catabolism via Prodh supports ATP production in spheroids, we performed a dynamic metabolomics experiment in MCF10A H-Ras ${ }^{\mathrm{V} 12}$ spheroids upon acute inhibition of Prodh activity using L-THFA. Interestingly, inhibition of Prodh activity by L-THFA significantly decreased ATP levels in MCF10A H-Ras ${ }^{V 12}$ spheroids $(P \leq 0.05$, two-tailed unpaired Student's $T$-test) (Fig. 4a). Because ATP production via Prodh requires the activity of complex III of the electron transport chain, but not that of complex I (Fig. 4b), we hypothesized that spheroidal growth of MCF10A H-Ras ${ }^{\mathrm{V} 12}$ is more susceptible to inhibition of complex III than of complex I. In agreement, we found that complex III inhibitor antimycin A, but not the complex I inhibitor rotenone, impaired spheroidal growth of MCF10A H-Ras V12 cells (Fig. 4c). Collectively, our data show that Prodh activity supports spheroidal growth by sustaining ATP production.

Prodh activity is specifically relevant in transformed cells. Our data suggest that Prodh may constitute a promising drug target against metastasis formation, especially if its activity would be 
a

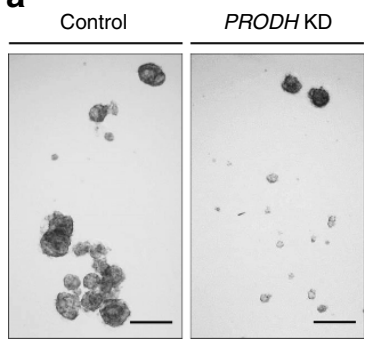

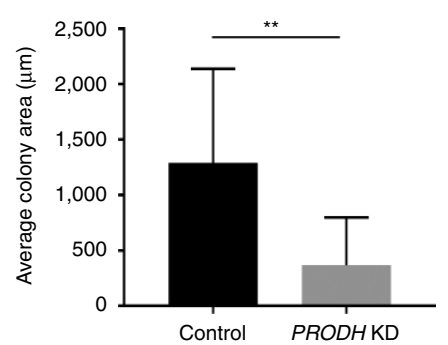

b

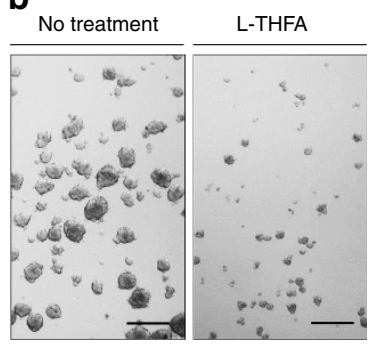

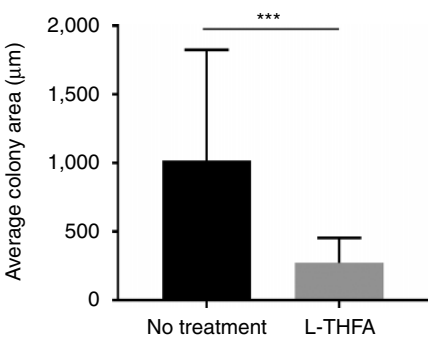

Figure 2 | Proline catabolism via Prodh supports 3D growth. (a) Representative pictures and size quantification of MCF10A H-Ras V12 spheroids transduced with an inducible lentiviral CRISPR with or without guide for PRODH. CRISPR expression was induced with $0.1 \mu \mathrm{g} \mathrm{ml} \mathrm{I}^{-1}$ doxycycline. Analysis was performed 5 days after PRODH knockdown (KD) induction. (b) Representative pictures and size quantification of MCF10A H-Ras ${ }^{V 12}$ spheroids with or without L-THFA treatment. Treatment was started at day 0. Analysis was performed at day 5 of treatment. Scale bar: $150 \mu \mathrm{m}$. The number of biological replicates for each experiment was $n \geq 3$. All error bars represent s.d. Two-tailed unpaired Student's $T$-test was performed. ${ }^{\star \star} P \leq 0.01 ;{ }^{\star \star \star} P \leq 0.001$.

specifically relevant for growth of transformed cells, but not of normal cells.

Thus, we tested whether PRODH expression was increased in transformed breast cancer cells during spheroidal growth. We measured the expression of $P R O D H$ in cell lines isolated from human or murine breast cancers (MCF7, HCC70, MDA-MB-231 and 4T1) during spheroidal (3D) compared to attached (2D) growth. We found that in all tested breast cancer cell lines PRODH expression increased during spheroidal growth (Fig. 5a). Moreover, we confirmed that 4T1 and EMT6.5 breast cancer cell lines, which we used for subsequent in vivo studies, increased proline uptake and were impaired by L-THFA treatment during spheroidal growth (Fig. 5b). This suggests that Prodh is relevant for spheroidal growth in breast cancer cell lines beyond MCF10A $\mathrm{H}-\mathrm{Ras} \mathrm{V} 12$.

Next, we tested whether Prodh activity is required for acinus formation of non-transformed MCF10A cells, which is a normal, non-invasive form of spheroidal growth ${ }^{29}$. Since non-transformed MCF10A cells fail to form acini without matrix attachment, we added $0.5 \%$ Matrigel to the DMEM-F12 media. We ruled out that Matrigel addition significantly changed organic and amino acid metabolism as well as growth upon Prodh inhibition in MCF10A H-Ras ${ }^{V 12}$ spheroids $(P>0.05$, two-tailed unpaired Student's $T$-test; Supplementary Fig. 5). Interestingly, we found that $P R O D H$ expression and ATP levels were significantly lower in MCF10A acini compared to MCF10A H-Ras V12 spheroids $(P \leq 0.05$, two-tailed unpaired Student's $T$-test; Fig. 5c,d), suggesting that Prodh activity is not required in non-transformed cells. In support of this notion, Prodh inhibition by L-THFA did neither impair acini formation nor reduce ATP levels of MCF10A cells (Fig. 5e,f). Similarly, inhibition of complex III or complex I had no effect on acini formation (Fig. 5g). Together, these data show that Prodh activity in general and ATP production via Prodh in particular are not required during normal acini formation of non-transformed mammary epithelial cells.

Prodh activity is increased in metastases tissue. Our in vitro data suggest that Prodh may be a drug target against metastasis formation, which is supported by the fact that $P R O D H$ is significantly higher expressed in metastases compared to primary breast cancers in patients $(P \leq 0.01$, two-tailed unpaired Student's $T$-test with Welchs correction; Fig. 6a). To test whether proline catabolism is indeed relevant for metastasis formation, we used an orthotopic 4T1 breast cancer mouse model. This model allowed us to investigate primary tumours and lung metastases within the same animal. As readout for Prodh activity, we measured enrichment of proline from ${ }^{13} \mathrm{C}_{6}$-glucose and proline levels in 4T1 primary breast cancers and the corresponding lung metastases using in vivo ${ }^{13} \mathrm{C}$ tracer analysis. This analysis revealed that both parameters were reduced in lung metastases as compared to the primary breast cancer tumours (Fig. 6b,c), which is consistent with increased proline catabolism in metastases tissue.

We next assessed potential adverse effects of the Prodh inhibitor L-THFA in mice. Since L-THFA was, to our knowledge, never applied in vivo, we measured alterations in metabolite levels in MCF10A H-Ras ${ }^{\mathrm{V} 12}$ spheroids upon treatment with L-THFA to define which metabolic changes need to be monitored in vivo to exclude adverse effects on healthy tissue. We found that mainly proline, alanine, glutamate, fumarate and malate levels were significantly changed upon treatment with L-THFA $(P \leq 0.05$, two-tailed unpaired Student's T-test; Fig. 6d). Since Prodh is physiologically expressed at high levels in the brain, heart and liver (human protein atlas), we measured the levels of the five metabolites in the brain, heart and liver of $\mathrm{BALB} / \mathrm{c}$ mice treated with increasing doses of L-THFA $\left(30,45,60 \mathrm{mg} \mathrm{kg}^{-1}\right)$. The compound was administered daily via intraperitoneal injections and metabolite levels were measured at day 16 of treatment. We found that only the lowest tested dose of L-THFA treatment did not result in a substantial increase in proline levels in these organs (Fig. 6e). Moreover, at this dose only minor and non-significant changes in alanine, glutamate, fumarate and malate levels were observed $(P>0.05$, two-tailed Student's $T$-test with $F$-testing to confirm equal variance).

To investigate the adverse effects beyond the level of central metabolism, we performed a full plasma/blood analysis and histopathology on $\mathrm{BALB} / \mathrm{c}$ mice injected for 16 days with $30 \mathrm{mg} \mathrm{kg}^{-1}$ L-THFA. This analysis showed that L-THFA treatment at this dose did not result in significant changes in haematology, clinical chemistry and organ histopathology compared to vehicle treatment of $\mathrm{BALB} / \mathrm{c}$ mice $(P>0.05$, two-tailed Student's $T$-test with $F$-testing to confirm equal variance; Supplementary Fig. 6). Based on this analysis, we decided to treat mice for all consecutive experiments with an L-THFA dose of $\leq 30 \mathrm{mg} \mathrm{kg}^{-1}$, which allowed safe administration for at least 16 days without obvious adverse effects.

Prodh inhibition impairs lung metastases formation. Finally, we tested whether inhibition of Prodh activity is sufficient to impair in vivo metastasis formation in the $4 \mathrm{~T} 1$ orthotopic breast cancer mouse model ${ }^{30}$. We induced primary tumour growth by injection of 4T1 breast cancer cells to the mammary fat pad of 6-week-old $\mathrm{BALB} / \mathrm{c}$ mice. After 4 days when the primary tumour nodule was established and palpable, we started daily intraperitoneal injections of L-THFA at $\leq 30 \mathrm{mg} \mathrm{kg}^{-1}$. Sixteen days after treatment, we analysed proline levels (to confirm Prodh 
a

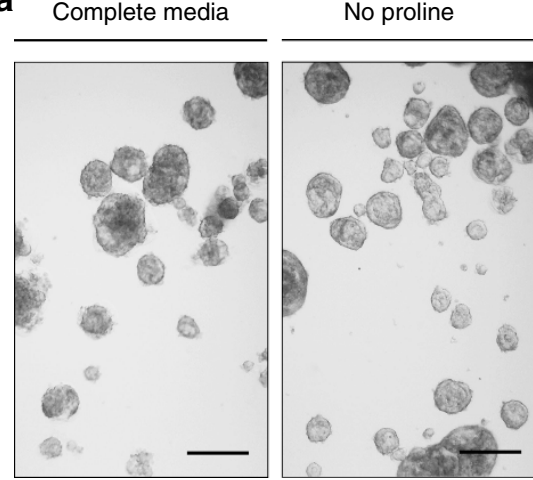

C
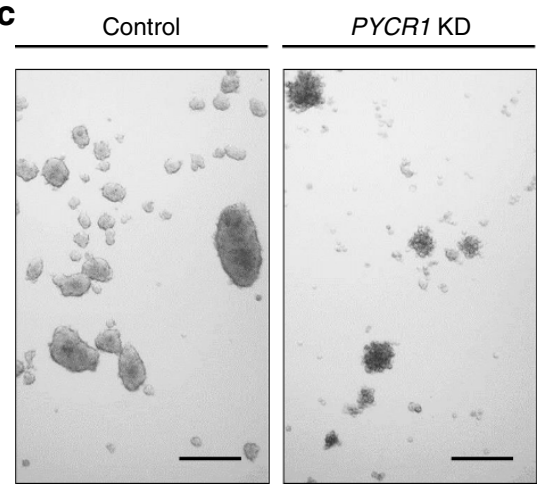

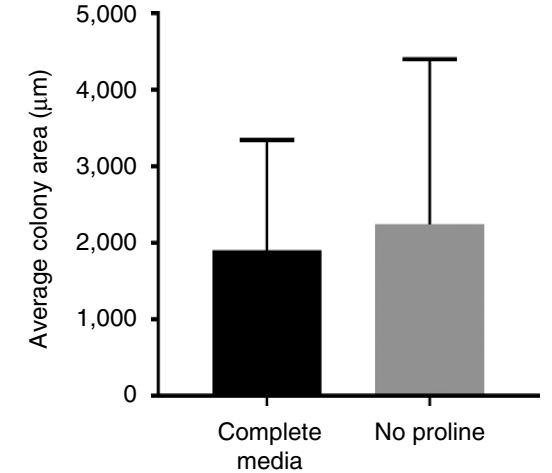

d

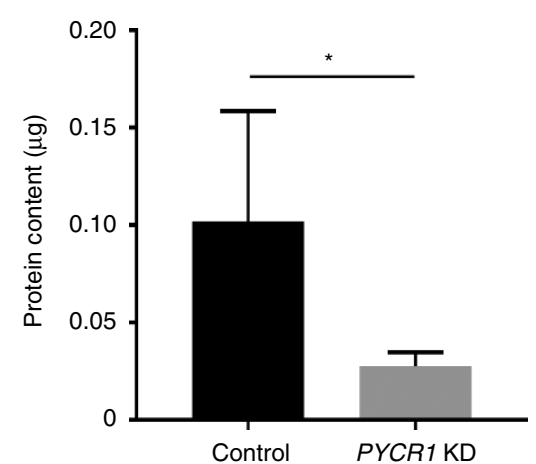

b

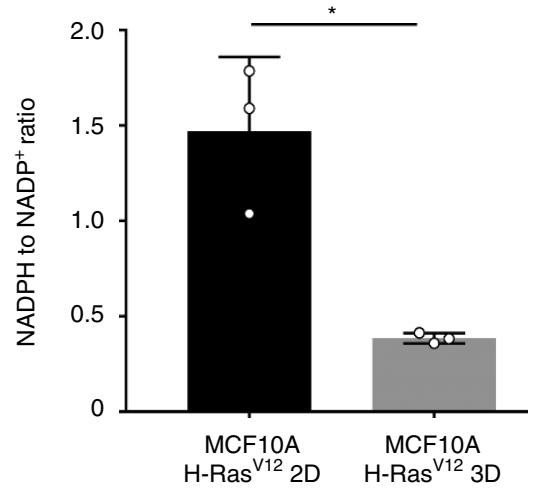

e

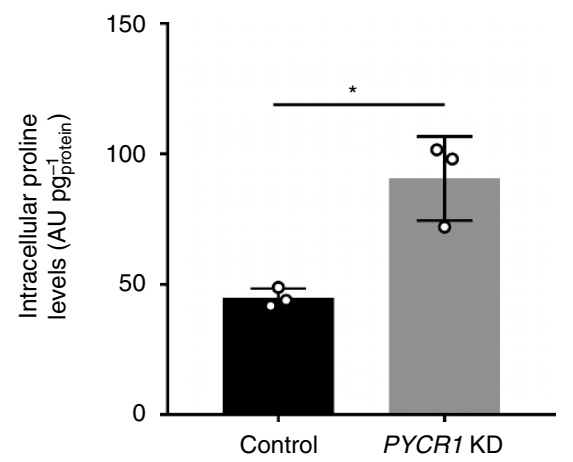

Figure 3 | Prodh activity depends on P5C recycling via Pycr1. (a) Representative pictures and size quantification of MCF10A H-Ras $\mathrm{V} 12$ spheroids in complete media and media without proline. Analysis was performed at day 5. Scale bar: $150 \mu \mathrm{m}$. (b) NADPH to NADP ${ }^{+}$ratio in MCF10A H-Ras ${ }^{\mathrm{V} 12}$ spheroids (3D) versus attached (2D) cells. (c) Representative pictures of MCF10A H-Ras ${ }^{\text {V12 }}$ spheroids transduced with a lentiviral vector with shRNA for either PYCR1 or a scrambled sequence. Analysis was performed at day 5. Scale bar: $150 \mu \mathrm{m}$. (d) Protein content in MCF10A H-Ras ${ }^{\mathrm{V} 12}$ spheroids transduced with a lentiviral vector with shRNA for either PYCR1 (KD) or a scrambled sequence. Analysis was performed at day 5. (e) Intracellular levels of proline in MCF10A H-Ras V12 spheroids transduced with a lentiviral vector with shRNA for either PYCR1 (KD) or scrambled sequence. Analysis was performed at day 5. Total ion counts were normalized for protein content. The number of biological replicates for each experiment was $n \geq 3$. All error bars represent s.d. Two-tailed unpaired Student's $T$-test was performed. ${ }^{\star} P \leq 0.05$.

inhibition in the metastases) and the number of metastases in the lungs as well as the primary breast tumour weight.

We found that proline levels were lower in the metastasis tissue compared to the primary breast cancer tissue only in animals treated with vehicle (Fig. 6c). Upon L-THFA treatment, this difference was not present anymore (Fig. 6c), which showed that L-THFA treatment resulted in the expected inhibition of Prodh activity within the metastasis tissue.

Strikingly, the weight of the primary breast cancer tumours remained unchanged upon L-THFA treatment (Fig. 7a), whereas the number of surface lung metastases decreased in a dose-dependent manner, with the strongest reduction in metastases $(\sim 60 \%)$ at $30 \mathrm{mg}$ $\mathrm{kg}^{-1}$ (Fig. 7b,c). Next, we performed haematoxylin and eosin (H\&E) staining of the lungs from animals treated with this dose, which confirmed the significantly reduced total number of metastases per lung section by approximately 50\% $(P \leq 0.05$, two-tailed Student's T-test with Welch's correction) (Fig. 7d,e). Finally, we validated the effect of Prodh inhibition on metastasis formation in the orthotopic EMT6.5 breast cancer mouse model ${ }^{30,31}$ (Fig. 7f-h).

In summary, our data demonstrate that Prodh inhibition is sufficient to impair formation of breast cancer-derived lung metastases.

\section{Discussion}

Here we show that proline catabolism via Prodh distinguishes breast cancer cells growing in spheroids (3D) from those growing in attached (2D) conditions. Inhibiting Prodh activity specifically impairs the capacity of breast cancer cells to form spheroids in vitro and lung metastases in vivo. Treatment with the Prodh inhibitor L-THFA had no adverse effects on non-transformed cells in vitro and in vivo. Thus, our data suggest Prodh as a promising drug target for preventing lung metastasis formation in breast cancer.

Unlike many other non-essential amino acids, the catabolism and biosynthesis of proline is catalysed by two different enzymes, namely Prodh and Pycr, that are coupled to two different co-factors. Thus, various roles of proline metabolism in transformed and non-transformed cells are possible. Indeed, inhibition of proline biosynthesis has been linked to unresolved endoplasmic reticulum stress in yeast and in cancer cells ${ }^{27,32}$. Moreover, it has been shown that proline biosynthesis can contribute to sustaining intracellular nucleotide levels ${ }^{26}$. In certain cancer cells, proline biosynthesis inhibition was found to impair tumorigenic potential and to decrease attached monolayer growth ${ }^{26,27}$. Yet, inhibition of proline catabolism in attached monolayer cultures resulted across different in vitro studies in both anti- and pro-growth/survival effects $^{28,33-39}$. Moreover, the in vivo role of Prodh activity in cancers has so far not been studied. This is challenging for advancing Prodh as a drug target, since it remains unclear under which in vivo conditions Prodh can be targeted to counteract cancer. We discovered that Prodh activity specifically impairs in vitro spheroidal growth and in vivo metastasis formation of 
a

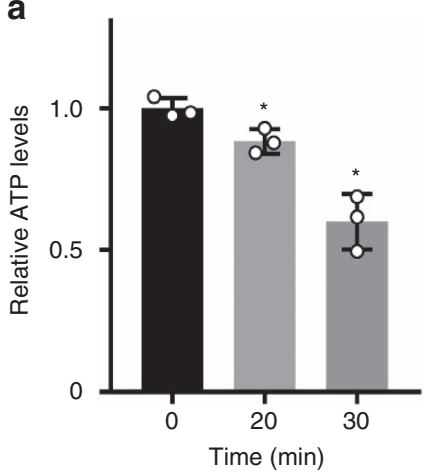

b

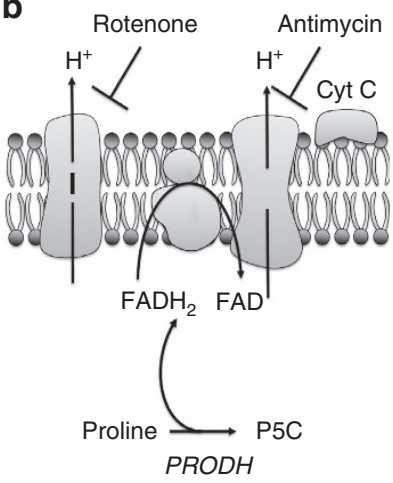

C
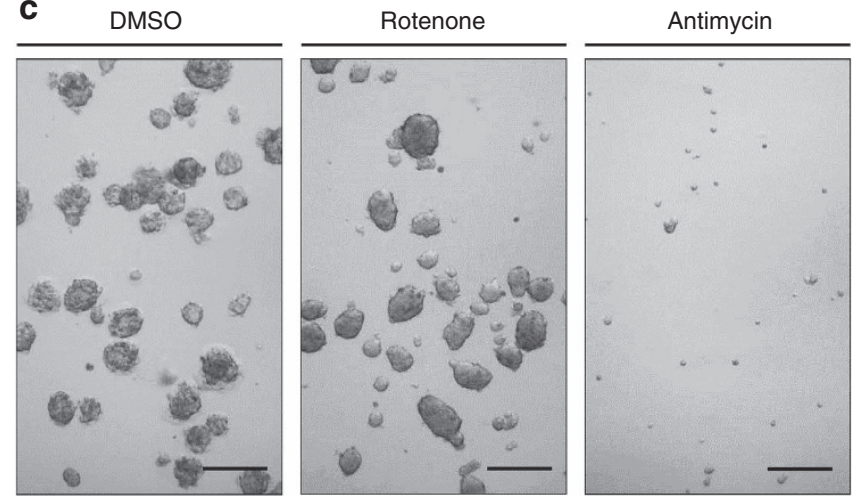

Figure 4 | Prodh activity generates ATP during 3D growth. (a) Timeresolved ATP levels of MCF10A H-Ras ${ }^{\mathrm{V} 12}$ spheroids upon treatment with the Prodh inhibitor L-THFA. (b) Schematic representation of Rotenone and Antimycin A mode of action as well as ATP generation from proline catabolism. (c) Representative pictures of MCF10A H-Ras ${ }^{\mathrm{V} 12}$ spheroids upon treatment with either rotenone or antimycin A. Treatment was started at day 0 . Analysis was performed at day 5 of treatment. Scale bar: $150 \mu \mathrm{m}$. The number of biological replicates for each experiment was $n \geq 3$. All error bars represent s.d. Two-tailed unpaired Student's $T$-test was performed. ${ }^{*} P \leq 0.05$.

breast cancer cells. Moreover, we show that treatment with the Prodh inhibitor L-THFA does not result in any adverse effects in normal cells and organ function (evaluated by organ metabolomics, haematology, clinical chemistry and organ histopathology) in mice. Thus, our study highlights Prodh as promising and likely safe therapeutic target for preventing breast cancer-derived metastasis formation.

In contrast to our recent finding that pyruvate carboxylasedependent anaplerosis distinguishes the metabolism of primary breast cancers from that of the resulting lung metastases due to the nutrient microenvironment ${ }^{13,40,41}$, the data presented here indicate that the dependence of lung metastases on Prodh activity is driven by shifts in the cellular phenotype. In accordance, proline catabolism seems to be specifically important during early stages of metastatic growth but less relevant in established secondary tumours. Consistent with this notion, we found that $P R O D H$ expression inversely correlated with spheroid size (Supplementary Fig. 7a) and that L-THFA treatment started after spheroid formation did not impair spheroid growth any longer (Supplementary Fig. 7b). Also, these data are consistent with the recent finding that MYC, which negatively regulates $P R O D H$ expression ${ }^{34}$, shows lower expression in micrometastases (defined by signatures of quiescent and dormancy genes) than macrometastases ${ }^{42}$. Consistently, we found that MYC expression is inversely correlated with $P R O D H$ expression in primary breast cancer tissue compared to metastases tissue of patients (Fig. 6a and Supplementary Fig. 7c).
The ability of cancer cells to perform phenotypic shifts is only one aspect of metastasis formation, and multiple other factors, including the extracellular matrix composition and stiffness, are important additional factors for metastasis formation ${ }^{43,44}$. Hence, it is tempting to speculate that the need of breast cancer cells for proline catabolism may depend on specific site/organ of metastasis. However, the finding that MYC, which negatively regulates $P R O D H$ expression, clusters with metastasis size rather than with the metastatic site ${ }^{42}$, suggests that Prodh inhibition may be independent of the organ in which metastases form.

Our data suggest that targeting Prodh activity could have the potential to be effective against dormant breast cancer cells and micrometastases. Notably, targeting such cancer cells is of specific interest for breast cancer treatment, because infiltration of distant organs leading to micrometastases formation is a very early event that often occurs before the primary tumour is resected $^{10,11}$ and which results years later in established secondary tumours and $90 \%$ of breast cancer deaths ${ }^{2}$. In summary, we identify Prodh as promising drug target against breast cancer-derived metastasis formation.

\section{Methods}

Cell culture. We generated MCF10A cells expressing H-Ras ${ }^{\mathrm{V} 12}$ (MCF10A $\mathrm{H}-\mathrm{Ras}^{\mathrm{V} 12}$ ) as well as control cells expressing an empty pLA vector (MCF10A) (Supplementary Fig. 1a). MCF10A and MCF10A H-Ras ${ }^{\mathrm{V} 12}$ cells were grown in DMEM-F12 supplemented with $5 \%$ horse serum, $1 \%$ penicillin $\left(50\right.$ units $^{-1}$ ), $1 \%$ streptomycin $\left(50 \mu \mathrm{g} \mathrm{ml}^{-1}\right), 0.5 \mathrm{\mu g} \mathrm{ml}^{-1}$ hydrocortisone, $100 \mathrm{ng} \mathrm{ml}^{-1}$ cholera toxin, $10 \mu \mathrm{g} \mathrm{ml}^{-1}$ insulin and $20 \mathrm{ng} \mathrm{ml}^{-1}$ recombinant human EGF. MCF7, HCC70 and MDA-MB-231 cells were cultured in DMEM supplemented with $10 \%$ fetal bovine serum, $1 \%$ penicillin $\left(50\right.$ units $\left.\mathrm{ml}^{-1}\right), 1 \%$ streptomycin $\left(50 \mu \mathrm{g} \mathrm{ml}^{-1}\right)$ and $0.15 \mathrm{mM}$ proline. $4 \mathrm{~T} 1$ cells were cultured in Roswell Park Memorial Institute medium with pyruvate supplement, $10 \%$ fetal bovine serum,

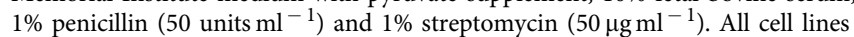
were confirmed to be mycoplasma-free by the Mycoalert Detection Kit (Lonza).

MCF10A, MCF7, HCC70, MDA-MB-231 and 4T1 cell lines were purchased from ATCC. All human cell lines were validated by DNA fingerprinting. The EMT6.5 cell line is a single-cell clone from the EMT6 cell line available from ATCC $^{31}$ and kindly provided by Professor Robin Anderson (Peter MacCallum Cancer Centre). None of the cell lines used for this study are listed in the database of commonly misidentified cell lines maintained by ICLAC. Both the 4T1 and EMT6 cell line-based orthotopic mouse models are widely used and accepted models for metastasis research ${ }^{30}$. Primary breast cancer of these models spontaneously metastasize within a short time frame and with a $100 \%$ penetrance

Two different 3D growth conditions were tested (Supplementary Fig. 1): For soft-agar culture, a base layer of agar was prepared in six-well plates. Soft-agar was dissolved at $1 \%$ in sterile distilled water in a microwave oven and was mixed with prewarmed $\left(37^{\circ} \mathrm{C}\right)$ medium at a ratio of $1: 1$. The base layer of agar mixture was transferred into a six-well plate at $3 \mathrm{ml}$ per well. Soft-agar was left to solidify at room temperature. Reduced growth factor Matrigel $(0.5 \%)$ without phenol red was added into the cold DMEM-F12 media before the seeding by using tips precooled to $-20^{\circ} \mathrm{C}$. MCF10A cells were plated on top of the base agar at 15,000 cells per well. For ultra-low attachment, cells were suspended sparsely and plated at 15,000 cells per well in six-well ultra-low Corning attachment plates. Unless otherwise noted, MCF10A H-Ras V12 were cultured on soft-agar without Matrigel addition. For ${ }^{13} \mathrm{C}$ carbon incorporation from glucose (Cambridge Isotope Laboratories, 99\%) and glutamine (Sigma, 98\%), cells were incubated for 5 days with labelled substrates. All analyses were performed after 5 days of incubation at $37^{\circ} \mathrm{C}$ in a $5 \% \mathrm{CO}_{2}$ incubator.

L-THFA was purchased from Sigma-Aldrich and used at the concentration of $5 \mathrm{mM}$ (MCF10A, MCF10A H-Ras V12 and 4T1 cells) and $10 \mathrm{mM}$ (EMT6.5 cells). The compound was added at day 0 and pictures were taken at day 5. For ATP analysis, L-THFA was used at day 5 for 0,20 and $30 \mathrm{~min}$ at the concentration of $10 \mathrm{mM}$. Rotenone and Antimycin A were purchased from Sigma-Aldrich and used at the concentration of 5 and $2 \mu \mathrm{M}$, respectively. The compounds were added at day 0 and pictures were taken at day 5 . All growth experiments were performed in $n \geq 3$ biological replicates.

shRNA knockdowns. The lentiviral pLKO shPYCR1 (NM_153824.1-398s1c1), shPYCR2 (NM_013328.2-996s1c1), shPYCR3 (NM_023078.x-1629s1c1) and scrambled control were purchased from the Broad Institute (Supplementary Table 1).

CRISPR interference knockdowns. The lentiviral CRISPR interference plasmid pHR-SFFV-KRAB-dCas9-P2A-mCherry was obtained from AddGene (Plasmid \#60954). Inducibility was achieved by subcloning the KRAB-dCas9-P2A-mcherry behind the TetO promoter of the lentiviral pTripZ vector (Open Biosystems RHS4750) through Gibson cloning. The U6 promoter and tracer sequence were 
a

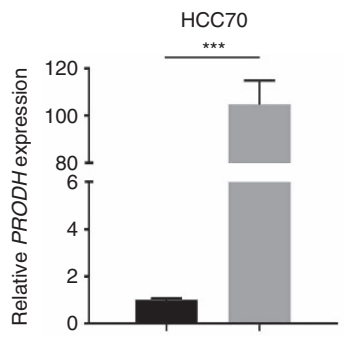

2D
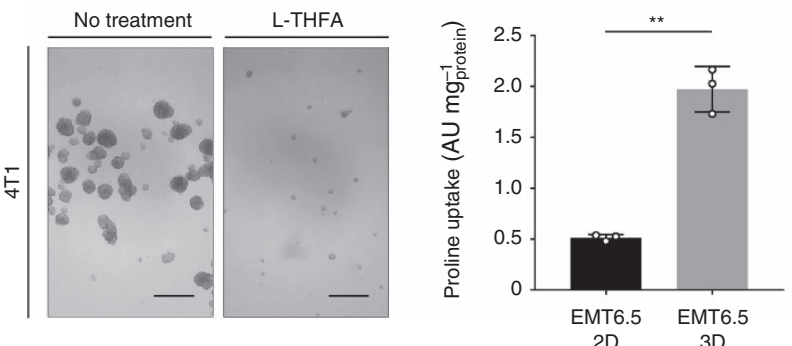

e

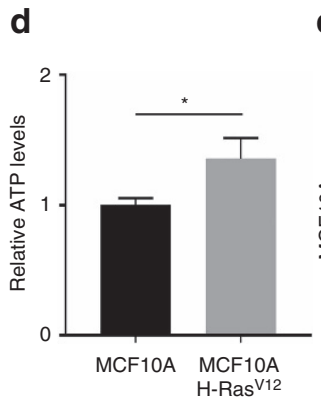

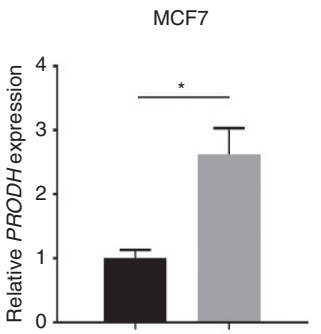

$2 \mathrm{D}$ f

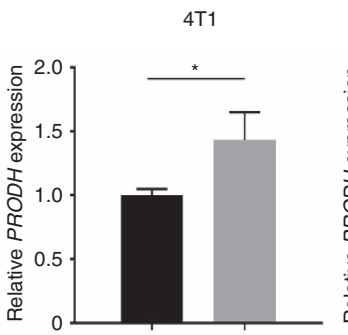

2D

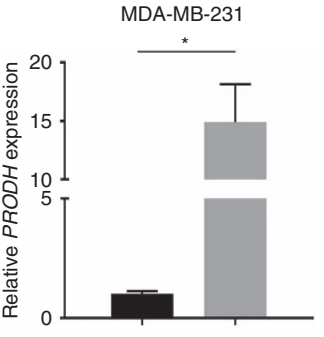

$2 \mathrm{D}$

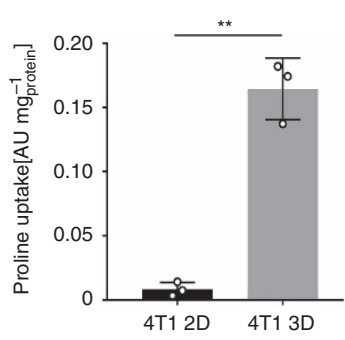

C

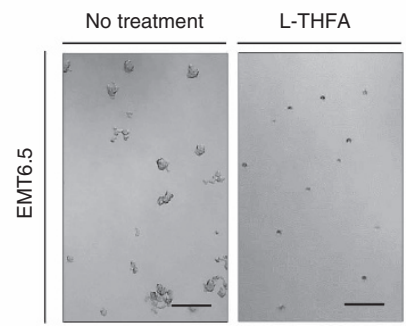

g

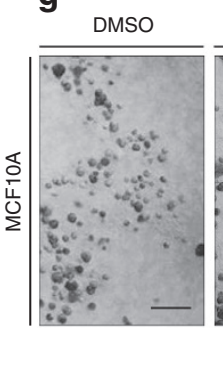

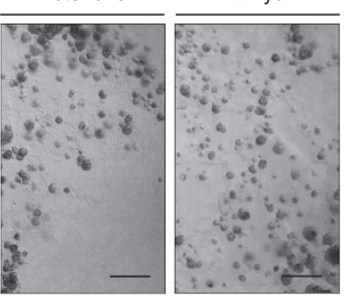

Figure 5 | Prodh activity is specifically relevant in transformed cells. (a) Relative expression of PRODH in HCC70, MCF7, 4T1 and MDA-MB-231 spheroids (3D) versus the corresponding attached (2D) cells. (b) Proline uptake of 4T1 and EMT6.5 spheroids versus attached cells and representative pictures of 4T1 and EMT6.5 spheroids with or without L-THFA treatment. Treatment was started at day 0 . Analysis was performed 5 days after treatment. Scale bar: $150 \mu \mathrm{m}$ (4T1 spheroids) and $75 \mu \mathrm{m}$ (EMT6.5 spheroids). (c) Relative expression of PRODH in MCF10A H-Ras $\mathrm{V12}$ spheroids compared to MCF10A acini. (d) Relative ATP levels of MCF10A H-Ras ${ }^{\mathrm{V} 12}$ spheroids compared to MCF10A acini. (e) Representative pictures of MCF10A acini with or without L-THFA treatment. Treatment was started at day 0. Analysis was performed 5 days after treatment. Scale bar: $150 \mu$ m. (f) Time-resolved ATP levels of MCF10A acini upon treatment with the Prodh inhibitor L-THFA. (g) Representative pictures of MCF10A acini upon treatment with either Rotenone or Antimycin A. Treatment was started at day 0. Analysis was performed 5 days after treatment. Scale bar: $150 \mu \mathrm{m}$. To each experiment, $0.5 \%$ Matrigel was added to the media of both cell lines to allow acini formation of non-transformed MCF10A cells and to enable the comparison between MCF10A H-Ras V12 and MCF10A cells. The number of biological replicates for each experiment was $n \geq 3$. All error bars represent s.d. Two-tailed unpaired Student's T-test was performed. ${ }^{\star} P \leq 0.05 ;{ }^{\star \star} P \leq 0.01 ;{ }^{\star \star \star} P \leq 0.001$.

cloned from pX330-U6-Chimeric-BB-CBh-hSpCas9, obtained from AddGene (Plasmid \#42230) through Gibson cloning. Guide specific for PRODH was selected by using the online guide design website available from http://crispr.mit.edu. From these, the top guide (GGCCCTGTAGTGCCGAAGCA) was selected and cloned $3^{\prime}$ to the U6 promoter through Gibson cloning ${ }^{45,46}$. Lentiviral particles were produced in HEK293 cells. MCF10A and MCF10A H-Ras V12 cells were selected with puromycin $1 \mu \mathrm{g} \mathrm{ml}^{-1}$. The expression of the CRISPR was induced with doxycycline $0.1 \mathrm{~g} \mathrm{ml}^{-1}$. As a control, an inducible CRISPR line lacking the guide for PRODH was generated.

Flow cytometry. In brief, MCF10A cells and MCF10A-derived colonies were lysed in trypsin $0.05 \%$ and $1 \times 10^{6}$ single cells were resuspended and incubated with antibodies at concentrations of $0.2 \mu \mathrm{g}$ per $100 \mu \mathrm{l}$ for CD24 conjugated with phycoerythrin (PE) (12-0247-42) and $0.1 \mu \mathrm{g}$ per $100 \mu \mathrm{l}$ for CD44 conjugated with PE-cyanine7 (Cy7) (560533), both purchased from BD Biosciences. Samples were stained in ice in the dark for $15 \mathrm{~min}$, and then the cells were washed twice with cold PBS, resuspended in $400 \mu \mathrm{l}$ PBS and analysed using a flow cytometer (BD FACS Verse). As negative controls, cells were treated with either isotype-matched control antibodies or with no primary antibody.
RNA isolation and quantitative real-time PCR. Total RNA was isolated with the PureLink RNA Mini Kit (Life Technologies). RNA (400 ng) was reverse transcribed into cDNA using a High-Capacity cDNA Reverse Transcription Kit (Life Technologies). The relative levels of gene transcripts compared to the control gene RPL-19 were determined by quantitative real-time PCR using SYBER Green PCR Master Mix (Life Technologies) and specific primers on a 7,500 Fast Real Time PCR System (Applied Biosystems, Life Technologies) (Supplementary Table 2). Amplification was performed at $95^{\circ} \mathrm{C}$ for $10 \mathrm{~min}$, followed by 40 cycles of $15 \mathrm{~s}$ at $95^{\circ} \mathrm{C}$ and $1 \mathrm{~min}$ at $60^{\circ} \mathrm{C}$. The fold change in gene expression was calculated as:

Fold change $=2^{-\Delta \Delta \mathrm{Ct}}$

Quenching and metabolite extraction methods. The quenching procedure applied was adapted from Sellick et al. ${ }^{47}$. Fifty-ml falcon tubes with methanol $60 \%$ and $10 \mathrm{mM}$ ammonium acetate were precooled in a dry ice-ethanol bath $\left(-40^{\circ} \mathrm{C}\right)$ for $20 \mathrm{~min}$. Colonies were transferred with a $1,000 \mu \mathrm{l}$ pipette from the soft-agar plates to $15 \mathrm{ml}$ falcon tubes and pelleted by centrifugation (1,400 r.p.m., $1 \mathrm{~min}$, room temperature) to remove excess of medium. In this step, four wells of one six-well plate were pooled into one falcon tube (one replicate). Medium was aspirated by using a vacuum line and the remaining cell pellet was immediately 
a

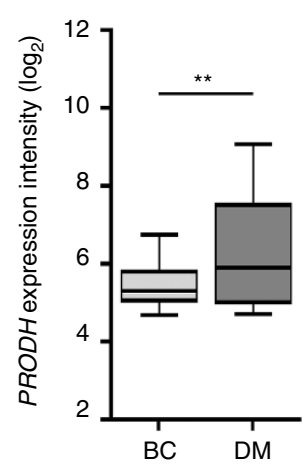

b

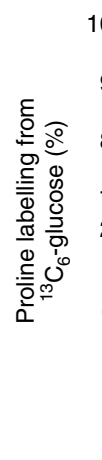

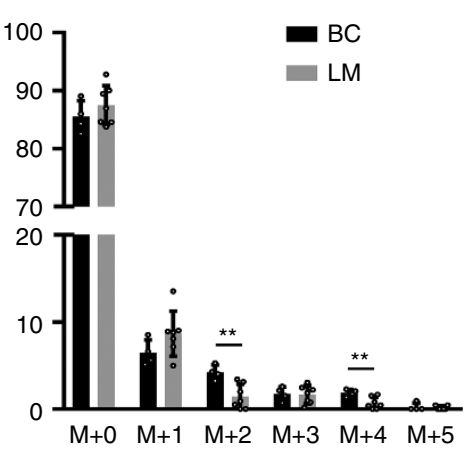

C

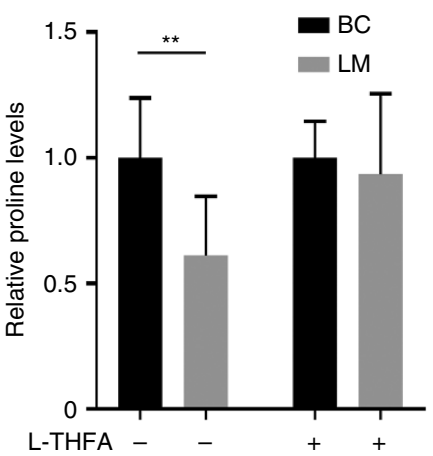

d

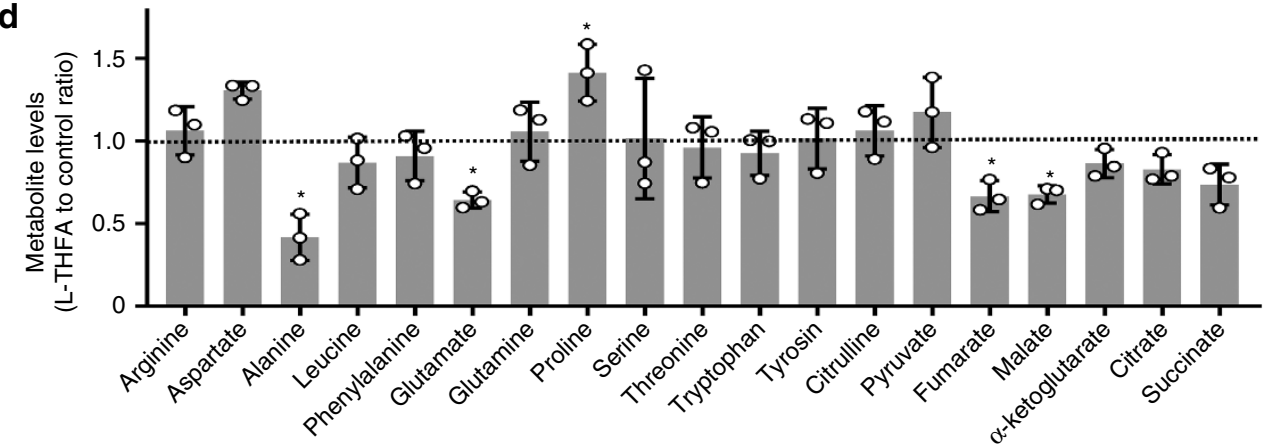

e

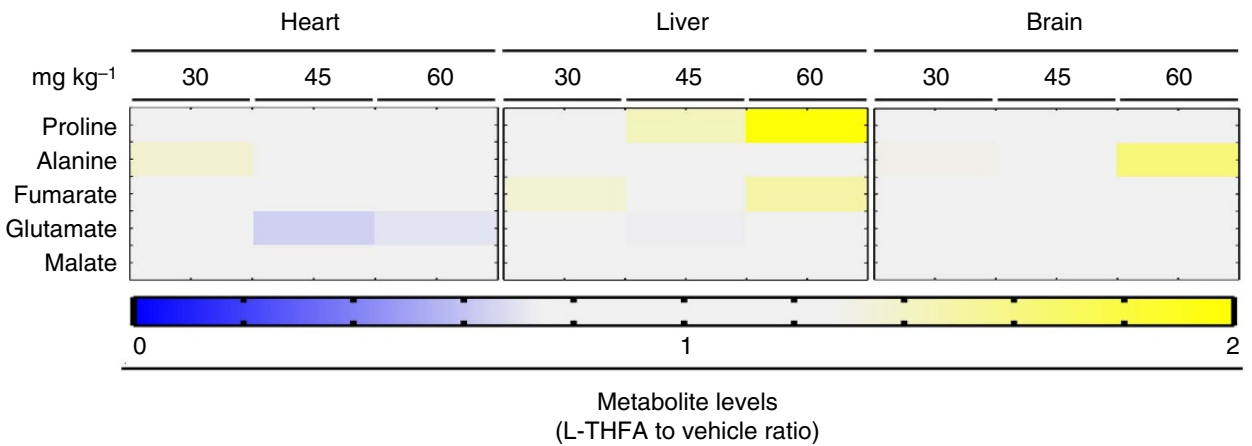

Figure 6 | Prodh activity is specifically relevant in metastasis tissue. (a) PRODH expression levels in primary breast cancer (BC) tissue (GSE20711) and breast cancer-derived metastases (DM) tissue (GSE14017) from patients. Data are shown as medians (bar) with the 25th-75th percentile range (box) and the 10th-90th percentile range (whiskers). Two-tailed unpaired Student's T-test with Welch's correction was performed. ${ }^{\star \star} P \leq 0.01$. (b) Proline labelling from ${ }^{13} \mathrm{C}_{6}$-glucose in primary breast cancers $(B C)$ and the resulting lung metastases (LM) of BALB/c mice orthotopically injected with murine $4 T 1$ breast cancer cells. Four mice with primary $B C$ and resulting $L M(n=7)$ were analysed. Two-tailed Student's $T$-test with $F$-testing to confirm equal variance was performed. ${ }^{\star \star} P \leq 0.01$. (c) Intracellular proline levels in primary breast cancers (BC) and the resulting lung metastases (LM) of BALB/C mice orthotopically injected with murine $4 \mathrm{~T} 1$ breast cancer cells and treated with either L-THFA or vehicle normalized to the respective control condition. For the vehicletreated group, $n=8$ mice with primary BC and resulting LM $(n=11)$ were analysed. For the L-THFA-treated group, $n=5$ mice with primary breast cancer and resulting LM $(n=5)$ were analysed. Two-tailed Student's $T$-test with $F$-testing to confirm equal variance was performed. ${ }^{\star \star} P \leq 0.01$. (d) Relative metabolite levels of MCF10A H-Ras ${ }^{\mathrm{V} 12}$ spheroids treated with or without L-THFA. Two-tailed unpaired Student's $T$-test was performed. ${ }^{*} P \leq 0.05$.

(e) Relative metabolite levels of the heart, liver and brain of mice treated with different doses of L-THFA compared to vehicle (PBS). Changes in metabolite levels $>27 \%$ are depicted $(n \geq 5)$. Two-tailed Student's T-test with $F$-testing to confirm equal variance was performed. Error bars represent s.d. unless otherwise noted.

added to the $50 \mathrm{ml}$ falcon tube containing $5 \mathrm{ml}$ of the quenching solution at $-40^{\circ} \mathrm{C}$. Cells were pelleted by centrifugation $(2,000$ r.p.m., 30 s, room temperature) and the supernatant was removed by quickly inverting the tube. The cell pellet was washed a second time with quenching solution $\left(-40^{\circ} \mathrm{C}\right)$. After the second wash, the cell pellet was stored at $-80^{\circ} \mathrm{C}$

Four different extractions methods were tested. All the extraction procedures were performed in a dry ice-ethanol bath $\left(-40^{\circ} \mathrm{C}\right)$. The protocols were adapted from the established published methods. The details of each procedure are described separately below. The methanol/chloroform extraction procedure was adapted from de Koning et al. ${ }^{19}$. The samples were resuspended in $800 \mu \mathrm{l}$ of precooled $60 \%$ methanol, before $500 \mu$ l of precooled chloroform were added Samples were vortexed for $10 \mathrm{~min}$ at $4{ }^{\circ} \mathrm{C}$ and then centrifuged (max. speed,
$10 \mathrm{~min}, 4^{\circ} \mathrm{C}$ ). The upper methanol/water phase, the intermediate protein layer and the lower chloroform phase were collected separately.

The hot ethanol extraction procedure was adapted from Bent et al. ${ }^{20}$. Ethanol $75 \%$ was preheated in a water bath at $80^{\circ} \mathrm{C}$ for $20 \mathrm{~min}$. Hot ethanol $(1 \mathrm{ml})$ was poured over the cell pellet. The samples were immediately vortexed and then placed in the water bath for $3 \mathrm{~min}$. After the $80^{\circ} \mathrm{C}$ incubation, the samples were placed back in the cold bath $\left(-40^{\circ} \mathrm{C}\right.$ ) and then centrifuged (max. speed, $5 \mathrm{~min}$, $4{ }^{\circ} \mathrm{C}$ ). The supernatants and the proteins were collected separately.

The trichloroacetic acid extraction procedure was adapted from Tunnicliffe et al. ${ }^{22}$. Samples were resuspended in precooled $5 \%$ trichloroacetic acid and then centrifuged (max. speed, $5 \mathrm{~min}, 4^{\circ} \mathrm{C}$ ). The supernatants and the protein were collected separately. 
a

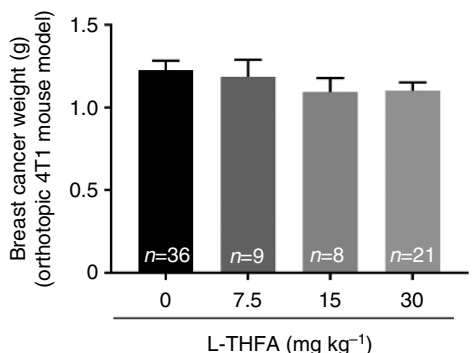

e

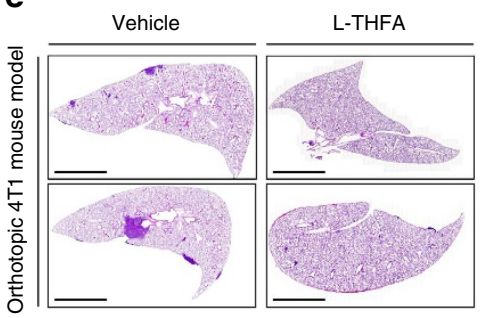

b

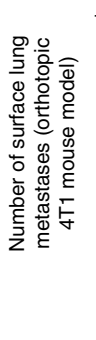

C

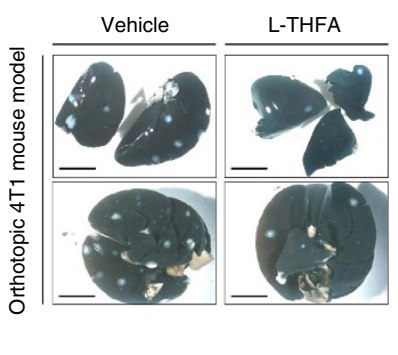

d

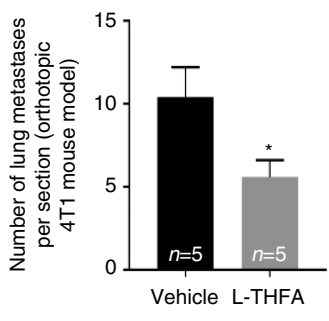

$\mathbf{f}$

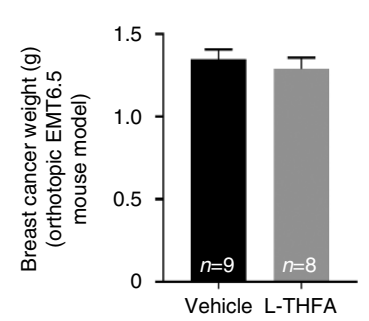

g

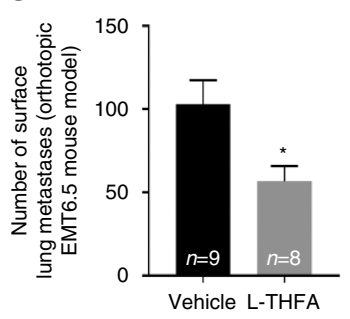

$\mathbf{h}$

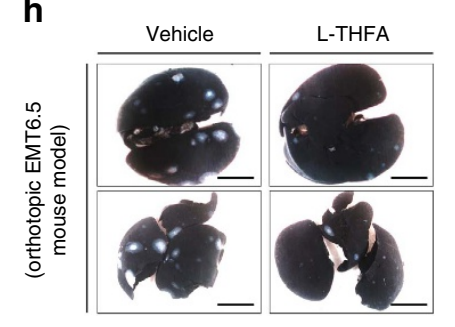

Figure 7 | Prodh inhibition impairs lung metastases formation. (a) Primary tumour weight, (b) the number of surface lung metastases and (c) representative pictures of ink-stained lungs with white tissue indicating metastases of BALB/C mice orthotopically injected with murine $4 \mathrm{T1}$ breast cancer cells treated with vehicle or L-THFA at different concentrations for 16 days: $0 \mathrm{mg} \mathrm{kg}^{-1}\left(n=36,4\right.$ independent cohorts), $7.5 \mathrm{mg} \mathrm{kg}{ }^{-1}$ ( $n=9$ ), $15 \mathrm{mg} \mathrm{kg}^{-1}(n=8)$, and $30 \mathrm{mg} \mathrm{kg}^{-1}(n=21,3$ independent cohorts). Treatment was started after primary breast tumours were formed. Scale bar: $0.5 \mathrm{~cm}$. Two-tailed unpaired Student's $T$-test with Welch's correction was performed. ${ }^{\star \star} P \leq 0.01$; ${ }^{\star \star \star} P \leq 0.001$. (d) Number of lung metastases per lung section and (e) representative pictures of $\mathrm{H} \& \mathrm{E}$-stained lung sections of BALB/c mice orthotopically injected with murine $4 \mathrm{~T} 1$ breast cancer cells treated with vehicle or L-THFA at $30 \mathrm{mg} \mathrm{kg}^{-1}$ ( $n=5$ per condition). Scale bar: $2.7 \mathrm{~mm}$. Two-tailed Student's $T$-test with $F$-testing to confirm equal variance was performed. ${ }^{\star} P \leq 0.05$. (f) Primary tumour weight, (g) the number of surface lung metastases and (h) representative pictures of ink-stained lungs (black) with metastases (white) of BALB/c mice orthotopically injected with murine EMT6.5 breast cancer cells treated with vehicle $(n=9)$ or L-THFA at 15 mg kg ${ }^{-1}$ $(n=8)$ for 18 days. Scale bar: $0.5 \mathrm{~cm}$. Two-tailed Student's $T$-test with $F$-testing to confirm equal variance was performed. ${ }^{\star} P \leq 0.05$. All error bars represent s.e.m.

The Acetonitrile-Methanol Extraction procedure was adapted from Rabinowitz et al..$^{21}$. Each sample was resuspended in $1 \mathrm{ml}$ of precooled acetonitrile/methanol/water (40:40:20) and centrifuged (max. speed, $5 \mathrm{~min}, 4^{\circ} \mathrm{C}$ ). The supernatants and the protein were collected separately.

By using a tissue lyser, a mechanical disruption step was performed after resuspension of the cell pellet with the chemicals of the respective extraction method at $-40^{\circ} \mathrm{C}$ for $5 \mathrm{~min}$.

The extracts were dried using a vacuum concentrator. Fatty acids were dried at $20^{\circ} \mathrm{C}$ for $1 \mathrm{~h}$, while polar metabolites were dried at $4{ }^{\circ} \mathrm{C}$ over night. The samples were stored at $-80^{\circ} \mathrm{C}$.

Gas chromatography-mass spectrometric analysis. Metabolites were derivatized and measured as described before ${ }^{48}$. In brief, polar metabolites were derivatized with $20 \mathrm{mg} \mathrm{ml}^{-1}$ methoxyamine in pyridine for $90 \mathrm{~min}$ at $37^{\circ} \mathrm{C}$ and subsequently with $\mathrm{N}$-(tert-butyldimethylsilyl)- $\mathrm{N}$-methyl-trifluoroacetamide, with $1 \%$ tert-butyldimethylchlorosilane for $60 \mathrm{~min}$ at $60^{\circ} \mathrm{C}$. Fatty acids were esterified with sulfuric acid/methanol for $72 \mathrm{~h}$ at $60^{\circ} \mathrm{C}$ and then extracted with hexane. Metabolites were measured with a 7890A GC system (Agilent Technologies) combined with a 5975C Inert MS system (Agilent Technologies). One microlitre of samples was injected in splitless mode with an inlet temperature of $270{ }^{\circ} \mathrm{C}$ onto a DB35MS column. The carrier gas was helium with a flow rate of $1 \mathrm{ml} \mathrm{min}^{-1}$. For the measurement of polar metabolites, the $\mathrm{GC}$ oven was held at $100^{\circ} \mathrm{C}$ for $3 \mathrm{~min}$ and then ramped to $300^{\circ} \mathrm{C}$ with a gradient of $2.5^{\circ} \mathrm{C} \mathrm{min}-1$. For the measurement of fatty acids, the oven was held at $80^{\circ} \mathrm{C}$ for $1 \mathrm{~min}$ and ramped with $5^{\circ} \mathrm{C} \mathrm{min}-1$ to $300^{\circ} \mathrm{C}$. The MS system was operated under electron impact ionization at $70 \mathrm{eV}$ and a mass range of 100-650 a.m.u. was scanned.

Mass distribution vectors were extracted from the raw ion chromatograms using a custom Matlab M-file, which applies consistent integration bounds and baseline correction to each ion. Moreover, we corrected for naturally occurring isotopes using the method of Fernandez et al. ${ }^{49}$. Total contribution of carbon was calculated using the following equation ${ }^{18}$ :

$$
\text { Total contribution of carbon }=\frac{\sum_{i=0}^{n} i \times m_{i}}{n \times \sum_{i=0}^{n} m_{i}}
$$

Here $n$ is the number of $\mathrm{C}$ atoms in the metabolite, $i$ the different mass isotopomers and $m$ the abundance of a certain mass. For metabolite levels, arbitrary units of the metabolites of interest were normalized to an internal standard and protein content (in vitro experiments) or tissue weight (in vivo experiments). In vivo, the
${ }^{13} \mathrm{C}$ enrichment of glucose in the blood of the mice was evaluated and confirmed to be similar between the infused animals.

Liquid chromatography-mass spectrometric analysis. Polar metabolites were resuspended in $60 \%$ acetonitrile. Metabolites were measured using a Dionex UtiMate 3,000 LC System (Thermo Scientific) combined with a Q Exactive Orbitrap mass spectrometer (Thermo Scientific) and run in negative mode, using mass spectrometry based on accurate mass. Samples were injected onto a SeQuant ZIC/pHILIC Polymeric column (Merck Millipore) ${ }^{50}$. The solvent, composed of acetonitrile and ammonium acetate $(\mathrm{pH}=9.3,10 \mathrm{mM})$, was used at a flow rate of $0.100 \mathrm{ml} \mathrm{min}^{-1}$. Data analysis was performed with the Xcalibur software. Metabolite levels were normalized to a fully ${ }^{13} \mathrm{C}$-labelled yeast extract and protein content. Alternatively, targeted measurements of polar metabolites were performed with a 1,290 Infinity II HPLC (Agilent) coupled to a 6,470 triple quadrupole mass spectrometer (Agilent). Samples were injected onto a iHILIC-Fusion $(\mathrm{P})$ column with the above-mentioned solvents. Data analysis was performed with the Agilent Mass Hunter software.

Analysis of gene expression microarray data. Microarray data of primary breast cancers (GSE20711) ${ }^{51}$ and breast cancer metastases (GSE14017) (52 $^{\text {generated on }}$ Affymetrix HG-U133Plus2.0 chips were retrieved from the Gene Expression Omnibus and normalized simultaneously by Robust Multichip Average ${ }^{53}$ using custom brain array chip description files (v18, ENTREZG) yielding one optimized probe-set per gen ${ }^{54}$. Statistical significance of differences in the gene expression levels were analysed with a two-tailed unpaired Student's T-test with Welch's correction using the GraphPad Prism 5 software (GraphPad Software Inc.).

In vivo ${ }^{13} \mathrm{C}$ tracer infusions. Six-week-old female $\mathrm{BALB} / \mathrm{c}$ mice were orthotopically inoculated with one million 4T1 cells. Primary tumour growth and body weight was monitored every second day. One week after tumour initiation, surgical catherization of the jugular vein was carried out as follows: BALB/c mice were anesthetized with $3 \%$ isoflurane with $2 \%$ oxygen and a catheter was placed into the right jugular vein. A small incision between shoulder blades was made and the catheter was exteriorized at the back of the mouse and connected to an antenna part, which enables conscious handling of the mouse during infusion. The tubing was flushed with heparinized saline directly after the surgery and 3 days later. Animals were individually housed after surgery, and painkillers were administered 
(Carprofen, $5 \mathrm{mg} \mathrm{kg}^{-1}$ body weight subcutaneously) during surgery and 1 day there after ${ }^{13,55,56}$

Two weeks after surgery, ${ }^{13} \mathrm{C}_{6}$-labelled glucose (99\% enriched; Sigma-Aldrich) infusions were performed $(n=4)$. Mice were subjected to a continuous infusion of $0.03 \mathrm{mg}{ }^{13} \mathrm{C}$-labelled glucose per gram body weight per min for the course of $6 \mathrm{~h}$ with a glucose solution of $500 \mathrm{mg}$ per $\mathrm{ml}{ }^{13} \mathrm{C}$-labelled glucose. Subsequently, mice were killed using nembutal, blood was collected and the breast tumours and lung metastases were placed in cold saline, dissected in $<3 \mathrm{~min}$ and immediately frozen using a liquid nitrogen cooled Biosqueezer (Biospec Products). The tissue was weighed $(10-15 \mathrm{mg})$ and pulverized (Cryomill, Retsch) under liquid nitrogen conditions. The pulverized tissue was extracted for GC-MS and LC-MS analysis as described above. Additionally, blood enrichment of ${ }^{13} \mathrm{C}$ glucose was analysed to ensure that no significant differences in blood enrichment per animal are observed. The animal study complies with ethical regulation and was approved by the $\mathrm{KU}$ Leuven ethics committee.

In vivo drug treatment. Six-week-old female $\mathrm{BALB} / \mathrm{c}$ mice were orthotopically inoculated with one million of either 4T1 or EMT6.5 cells. For inhibition of Prodh activity, L-THFA treatment was started after 4 days when the primary tumour nodule was established and palpable. Different doses of L-THFA $\left(60 \mathrm{mg} \mathrm{kg}^{-1}\right.$ $(n=6), 45 \mathrm{mg} \mathrm{kg}^{-1}(n=6), 30 \mathrm{mg} \mathrm{kg}^{-1}$ ( $n=21$ with 3 independent cohorts), $\left.15 \mathrm{mg} \mathrm{kg}^{-1}(n=8), 7.5 \mathrm{mg} \mathrm{kg}^{-1}(n=9)\right)$ were injected daily intraperitoneally to healthy or cancer-bearing mice. L-THFA was dissolved in PBS and $\mathrm{pH}$ neutralized with $\mathrm{NaOH}$. PBS was injected as a vehicle to control animals $(n=36$ with 4 independent cohorts). L-THFA-treated and control mice were randomly chosen. Mice were killed after 16 days (4T1 model) or 18 days (EMT6.5 model) of treatment. In case of cancer-bearing mice, lung metastases were visualized by intratracheal injection of ink solution (15\% of final volume of black ink, water, 2-3 drops ammonia). Black ink-injected lungs were washed in distaining solution $(100 \mathrm{ml} 70 \% \mathrm{EtOH}, 100 \mathrm{ml} 37 \%$ formaldehyde, $5 \mathrm{ml}$ glacial acetic acid) and then placed in fresh distaining solution overnight. Two different researchers in a blind manner counted white tumour nodules against a black lung background. The animal study complies with ethical regulation and was approved by the KU Leuven ethics committee.

In vivo histopathology analysis. A short-term toxicology study was carried out in order to rule out potential adverse effect/toxicity of the drug under the same treatment conditions used for the original efficacy study. Briefly, six-week-old $\mathrm{BALB} / \mathrm{c}$ females were randomly divided into two groups ( $n=5$ per group) and treated with $30 \mathrm{mg} \mathrm{kg}^{-1}$ of L-THFA (group I) or vehicle only (group II) once a day for 16 days via peritoneal injection. During the treatment period, the following clinical, physiological and behavioural parameters were constantly monitored and recorded: body weight, food and water consumption, body condition score, home cage activity, and locomotion. Twenty-four hours after the last injection, animals were killed by $\mathrm{CO}_{2}$ asphyxiation. Terminal blood collection was also performed via cardiac puncture. Next, the mice underwent complete necropsy with gross examination and organ weights. The following organs/tissues were finally sampled and immersion fixed for $48-72 \mathrm{~h}$ in $10 \%$ neutral buffered formalin: truncal skin, entire head, spine, salivary glands, esophagus, larynx, trachea, lungs, heart, gastrointestinal tract, liver and gall bladder, pancreas, kidneys, ovaries, uterus, urinary bladder, lymph nodes (cervical, mesenteric), spleen, thymus, left hindlimb, and sternum.

Formalin-fixed tissues samples were routinely processed and embedded in paraffin blocks for histopathological examination (Thermo Scientific Excelsior AS Tissue Processor and HistoStar Embedding Workstation). $5 \mu \mathrm{m}$ thick sections obtained from these blocks (Thermo Scientific Microm HM355S microtome) were then stained with H\&E (Leica ST5010 Autostainer XL) and evaluated under a Leica DM 2,500 light microscope by a board-certified veterinary pathologist. Trimming and orientation of the various organs/tissues collected were performed following the RITA guidelines (revised guides for organ sampling and trimming in rats and mice). Microscopic lesions observed during histopathological examination were classified according to the INHAND (International Harmonization of Nomenclature and Diagnostic Criteria) system. Histopathological examination was performed in a blind manner and details concerning experimental design and tested compounds were revealed only at the end of the study. Whole-blood samples were collected in BD Microtainer K2E tubes (ref. \#365955). Haematology was performed using the scil Vet abc Plus analyser. Clinical chemistry was conducted on plasma separated from the whole blood.

For the quantification of pulmonary metastasis, dissected lung samples were gently infused via the trachea with $10 \%$ neutral buffered formalin and then processed for histopathological examination as previously described. $5 \mu \mathrm{m}$ thick sections obtained from the resulting paraffin blocks were stained with H\&E. Reconstruction and absolute counts of metastatic lesions were finally performed on four step sections with an interval of $30 \mu \mathrm{m}$ and therefore spanning an overall parenchymal thickness of $110 \mu \mathrm{m}$. The animal study complies with ethical regulation and was approved by the KU Leuven ethics committee.

Statistical analysis. Two-tailed unpaired Student's $T$-tests were performed on $n \geq 3$ biological replicates. For the in vivo data, statistical significance was calculated by standard two-tailed Student's $T$-tests with $F$-testing to confirm equal variance. In case of unequal variance, Welch's correction was performed. Detection of mathematical outliers was performed using Grubb's test in GraphPad. Sample size for all experiments was chosen empirically. Independent experiments were pooled and analysed together whenever possible as detailed in figure legends. All graphs show mean \pm s.d. or s.e.m. as indicated in the figure legends.

Data availability. Microarray data of primary breast cancers (GSE20711) ${ }^{51}$ and breast cancer metastases (GSE14017) ${ }^{52}$ generated on Affymetrix HG-U133Plus2.0 chips are available through Gene Expression Omnibus. The authors declare that all the other data supporting the findings of this study are available within the article and its Supplementary Information files and from the corresponding author upon reasonable request.

\section{References}

1. Hanahan, D. \& Weinberg, R. A. Hallmarks of cancer: the next generation. Cell 144, 646-674 (2011)

2. Elia, I., Schmieder, R., Christen, S. \& Fendt, S. M. Organ-specific cancer metabolism and its potential for therapy. Handb. Exp. Pharmacol. 33, 321-353 (2016).

3. Valastyan, S. \& Weinberg, R. A. Tumor metastasis: molecular insights and evolving paradigms. Cell 147, 275-292 (2011).

4. Ferlay, J. et al. Cancer incidence and mortality worldwide: sources, methods and major patterns in GLOBOCAN 2012. Int. J. Cancer 136, E359-E386 (2015).

5. Giancotti, F. G. Mechanisms governing metastatic dormancy and reactivation. Cell 155, 750-764 (2013).

6. Fidler, I. J. The pathogenesis of cancer metastasis: the 'seed and soil' hypothesis revisited. Nat. Rev. Cancer 3, 453-458 (2003).

7. Piskounova, E. et al. Oxidative stress inhibits distant metastasis by human melanoma cells. Nature 527, 186-191 (2015)

8. Yang, L. et al. Metabolic shifts toward glutamine regulate tumor growth, invasion and bioenergetics in ovarian cancer. Mol. Syst. Biol. 10, 728 (2014).

9. Boroughs, L. K. \& DeBerardinis, R. J. Metabolic pathways promoting cancer cell survival and growth. Nat. Cell Biol. 17, 351-359 (2015).

10. Harper, K. L. et al. Mechanism of early dissemination and metastasis in Her2 + mammary cancer. Nature. doi:10.1038/nature20609 (2016).

11. Hosseini, H. et al. Early dissemination seeds metastasis in breast cancer. Nature doi:10.1038/nature20785 (2016).

12. Dupuy, F. et al. PDK1-dependent metabolic reprogramming dictates metastatic potential in breast cancer. Cell Metab. 22, 577-589 (2015).

13. Christen, S. et al. Breast cancer-derived lung metastases show increased pyruvate carboxylase-dependent anaplerosis. Cell Rep. 17, 837-848 (2016).

14. Hoenerhoff, M. J. et al. BMI1 cooperates with H-RAS to induce an aggressive breast cancer phenotype with brain metastases. Oncogene 28, 3022-3032 (2009).

15. Lock, R., Kenific, C. M., Leidal, A. M., Salas, E. \& Debnath, J. Autophagydependent production of secreted factors facilitates oncogenic RAS-driven invasion. Cancer Discov. 4, 466-479 (2014).

16. Soule, H. D. et al. Isolation and characterization of a spontaneously immortalized human breast epithelial cell line, MCF-10. Cancer Res. 50, 6075-6086 (1990).

17. von Lintig, F. C. et al. Ras activation in human breast cancer. Breast Cancer Res. Treat. 62, 51-62 (2000).

18. Buescher, J. M. et al. A roadmap for interpreting C metabolite labeling patterns from cells. Curr. Opin. Biotechnol. 34, 189-201 (2015).

19. de Koning, W. \& van Dam, K. A method for the determination of changes of glycolytic metabolites in yeast on a subsecond time scale using extraction at neutral pH. Anal. Biochem. 204, 118-123 (1992).

20. Bent, K. J. \& Morton, A. G. Amino acid composition of fungi during development in submerged culture. Biochem. J. 92, 260-269 (1964).

21. Rabinowitz, J. D. \& Kimball, E. Acidic acetonitrile for cellular metabolome extraction from Escherichia coli. Anal. Chem. 79, 6167-6173 (2007).

22. Tunnicliffe, H. E. Glutathione: the occurrence and quantitative estimation of glutathione in tissues. Biochem. J. 19, 194-198 (1925).

23. Grassian, A. R., Metallo, C. M., Coloff, J. L., Stephanopoulos, G. \& Brugge, J. S Erk regulation of pyruvate dehydrogenase flux through PDK4 modulates cell proliferation. Genes Dev. 25, 1716-1733 (2011).

24. Jiang, L. et al. Reductive carboxylation supports redox homeostasis during anchorage-independent growth. Nature 532, 255-258 (2016).

25. Krishnan, N., Dickman, M. B. \& Becker, D. F. Proline modulates the intracellular redox environment and protects mammalian cells against oxidative stress. Free Radic. Biol. Med. 44, 671-681 (2008).

26. Liu, W., Hancock, C. N., Fischer, J. W., Harman, M. \& Phang, J. M. Proline biosynthesis augments tumor cell growth and aerobic glycolysis: involvement of pyridine nucleotides. Sci. Rep. 5, 17206 (2015).

27. Sahu, N. et al. Proline starvation induces unresolved ER stress and hinders mTORC1-dependent tumorigenesis. Cell Metab. 24, 753-761 (2016). 
28. Pandhare, J., Donald, S. P., Cooper, S. K. \& Phang, J. M. Regulation and function of proline oxidase under nutrient stress. J. Cell Biochem. 107, 759-768 (2009).

29. Imbalzano, K. M., Tatarkova, I., Imbalzano, A. N. \& Nickerson, J. A. Increasingly transformed MCF-10A cells have a progressively tumor-like phenotype in three-dimensional basement membrane culture. Cancer Cell Int. 9, 7 (2009).

30. Khanna, C. \& Hunter, K. Modeling metastasis in vivo. Carcinogenesis 26, 513-523 (2005).

31. Song, K., Benhaga, N., Anderson, R. L. \& Khosravi-Far, R. Transduction of tumor necrosis factor-related apoptosis-inducing ligand into hematopoietic cells leads to inhibition of syngeneic tumor growth in vivo. Cancer Res. 66, 6304-6311 (2006)

32. Liang, X., Dickman, M. B. \& Becker, D. F. Proline biosynthesis is required for endoplasmic reticulum stress tolerance in Saccharomyces cerevisiae. J. Biol. Chem. 289, 27794-27806 (2014).

33. Natarajan, S. K. et al. Proline dehydrogenase is essential for proline protection against hydrogen peroxide-induced cell death. Free Radic. Biol. Med. 53, 1181-1191 (2012).

34. Liu, W. et al. Reprogramming of proline and glutamine metabolism contributes to the proliferative and metabolic responses regulated by oncogenic transcription factor c-MYC. Proc. Natl Acad. Sci. USA 109, 8983-8988 (2012).

35. Liu, Y. et al. MnSOD inhibits proline oxidase-induced apoptosis in colorectal cancer cells. Carcinogenesis 26, 1335-1342 (2005).

36. Liu, W. et al. Proline oxidase promotes tumor cell survival in hypoxic tumor microenvironments. Cancer Res. 72, 3677-3686 (2012).

37. Liu, Y. et al. Proline oxidase functions as a mitochondrial tumor suppressor in human cancers. Cancer Res. 69, 6414-6422 (2009).

38. Liu, W. et al. miR-23b targets proline oxidase, a novel tumor suppressor protein in renal cancer. Oncogene 29, 4914-4924 (2010).

39. Donald, S. P. et al. Proline oxidase, encoded by p53-induced gene-6, catalyzes the generation of proline-dependent reactive oxygen species. Cancer Res. 61, 1810-1815 (2001).

40. Elia, I. \& Fendt, S. M. In vivo cancer metabolism is defined by the nutrient microenvironment. Transl. Cancer Res. 5(Suppl 6): S1284-S1287 (2016).

41. Lorendeau, D., Christen, S., Rinaldi, G. \& Fendt, S. M. Metabolic control of signalling pathways and metabolic auto-regulation. Biol. Cell 107, 251-272 (2015).

42. Lawson, D. A. et al. Single-cell analysis reveals a stem-cell program in human metastatic breast cancer cells. Nature 526, 131-135 (2015).

43. Acerbi, I. et al. Human breast cancer invasion and aggression correlates with ECM stiffening and immune cell infiltration. Integr. Biol. (Camb.) 7, 1120-1134 (2015).

44. Fidler, I. J. et al. Correlation of growth capacity of cells in hard agarose with successful transfection by the activated c-Ha-ras oncogene and in vivo proliferative capacity at metastatic sites. Anticancer Res. 11, 17-24 (1991).

45. Mandegar, M. A. et al. CRISPR interference efficiently induces specific and reversible gene silencing in human iPSCs. Cell Stem Cell 18, 541-553 (2016).

46. Gilbert, L. A. et al. Genome-scale CRISPR-mediated control of gene repression and activation. Cell 159, 647-661 (2014).

47. Sellick, C. A., Hansen, R., Stephens, G. M., Goodacre, R. \& Dickson, A. J. Metabolite extraction from suspension-cultured mammalian cells for global metabolite profiling. Nat. Protoc. 6, 1241-1249 (2011).

48. Lorendeau, D. et al. Dual loss of succinate dehydrogenase (SDH) and complex I activity is necessary to recapitulate the metabolic phenotype of SDH mutant tumors. Metab. Eng. S1096-7176, 30217-30218 (2016).

49. Fernandez, C. A., Des Rosiers, C., Previs, S. F., David, F. \& Brunengraber, H. Correction of $13 \mathrm{C}$ mass isotopomer distributions for natural stable isotope abundance. J. Mass Spectrom. 31, 255-262 (1996).

50. Pluskal, T., Nakamura, T., Villar-Briones, A. \& Yanagida, M. Metabolic profiling of the fission yeast $S$. pombe: quantification of compounds under different temperatures and genetic perturbation. Mol. Biosyst. 6, 182-198 (2010).

51. Dedeurwaerder, S. et al. DNA methylation profiling reveals a predominant immune component in breast cancers. EMBO Mol. Med. 3, 726-741 (2011).

52. Xu, J. et al. 14-3-3zeta turns TGF-beta's function from tumor suppressor to metastasis promoter in breast cancer by contextual changes of Smad partners from p53 to Gli2. Cancer Cell 27, 177-192 (2015).
53. Irizarry, R. A. et al. Exploration, normalization, and summaries of high density oligonucleotide array probe level data. Biostatistics 4, 249-264 (2003).

54. Dai, M. et al. Evolving gene/transcript definitions significantly alter the interpretation of GeneChip data. Nucleic Acids Res. 33, e175 (2005).

55. Davidson, S. M. et al. Environment impacts the metabolic dependencies of Ras-driven non-small cell lung cancer. Cell Metab. 23, 517-528 (2016).

56. Quaegebeur, A. et al. Deletion or inhibition of the oxygen sensor PHD1 protects against ischemic stroke via reprogramming of neuronal metabolism. Cell Metab. 23, 280-291 (2016).

\section{Acknowledgements}

We thank Dr Pier Andrée Penttila from the KU Leuven FACS analysis core, Dr Stefan Vinckier from the VIB microscopy core, Dr Bart Ghesquière from the VIB Metabolomics core and Professor Massimiliano Mazzone (VIB Leuven) for their support. Professor Anna Sablina (VIB Leuven), Professor Peter Carmeliet (VIB Leuven), Professor John Blenis (Cornell Medical), Professor Robin Anderson (Peter MacCallum Cancer Centre) and Professor Daniel Tennant (University of Birmingham) provided reagents. Dr Roberta Schmieder (VIB Leuven) and Dr Shawn Davidson (MIT) helped with the setup of the in vivo infusion technology. The laboratory of TGPG is supported by grants from the 'Verein zur Förderung von Wissenschaft und Forschung an der Medizinischen Fakultät der LMU München (WiFoMed)', the Daimler and Benz Foundation in cooperation with the Reinhard Frank Foundation, by LMU Munich's Institutional Strategy LMUexcellent within the framework of the German Excellence Initiative, the 'Mehr LEBEN für krebskranke Kinder-Bettina-Bräu-Stiftung', the Walter Schulz Foundation, the Fritz Thyssen Foundation and the Wilhelm Sander-Foundation (2016.167.1), the Friedrich-Baur Foundation and by the German Cancer Aid (DKH111886 and DKH-70112257). S.M.F. acknowledges funding support from Marie CurieCIG, FWO-Odysseus II, Concern Foundation, FWO-Research Grants, Eugéne Yourassowsky Schenking, KU Leuven-Methusalem Co-Funding and Bayer Health Care (grants4targets).

\section{Author contributions}

I.E. performed all experiments and analysed the data. D.B. helped with mouse surgeries, in vivo ${ }^{13} \mathrm{C}$ tracer infusions and in vivo L-THFA experiments. S.C. supported the optimization of the extraction method. R.B. and C.V. gave advice for gene expression analysis and CRISPR knockdown. E.R. performed the haematology and organ histopathology analysis. M.F.O. and T.G.P.G. performed bioinformatic analyses on microarray data and provided statistical guidance. T.G.P.G. helped revising the final manuscript. I.E. and S.-M.F. designed the study and wrote the manuscript. S.-M.F. conceived and supervised the study.

\section{Additional information}

Supplementary Information accompanies this paper at http://www.nature.com/ naturecommunications

Competing interests: The authors declare no competing financial interests.

Reprints and permission information is available online at http://npg.nature.com/ reprintsandpermissions/

How to cite this article: Elia, I. et al. Proline metabolism supports metastasis formation and could be inhibited to selectively target metastasizing cancer cells. Nat. Commun. 8, 15267 doi: 10.1038/ncomms15267 (2017)

Publisher's note: Springer Nature remains neutral with regard to jurisdictional claims in published maps and institutional affiliations.

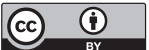

This work is licensed under a Creative Commons Attribution 4.0 International License. The images or other third party material in this article are included in the article's Creative Commons license, unless indicated otherwise in the credit line; if the material is not included under the Creative Commons license, users will need to obtain permission from the license holder to reproduce the material. To view a copy of this license, visit http://creativecommons.org/licenses/by/4.0/

C) The Author(s) 2017 